\title{
An Investigate on Sleep Quality in Elderly Inpatient and Its Influence Factor
}

\author{
Yanfeng Liu \\ Medical college of Yan'an University, Yan'an, Shaanxi, 716000
}

\begin{abstract}
Objective: The sleep quality and the influence factor of elderly inpatient is understood, the data of sleep quality and nursing interventions are provided; Method: 230 elderly inpatient (above 60 years oid) in geriatrics of Yan'an are selected by using the convenience sampling method in March to September 2015, the investigate on elderly inpatient of sleep quality and influence factor is carried out by the questionnaire survey (SRSS). Results: 263 questionnaires were handed out, which resulted in $87.8 \%$ (230) sample return, $96 \%$ of elderly patients have varying degrees of insomnia, the sleep problems are mainly after the reaction of insomnia, difficulty falling asleep, sleep instability, early awakening. the main factors of the reason of leading to insomnia in elderly patients are physiological discomfort caused by the pathological factors, psychological stress and the change of environmental and lifestyle. Conclusion: Insomnia of elderly patients have universality and particularity, so the medical staff should have enough attention to it, and actively symptomatic treatment, good health education and psychological care must be do to elderly patients, in order to reduce the rate of insomnia in elderly patients, and facilitate recovery from disease.
\end{abstract}

Keywords: Elderly patients; Sleep quality; Influence factor

\section{Introduction}

With the aging of our population increasingly deepened, the health of older people more and more attention [1], of which the elderly sleep disorders than other age there is a higher proportion, which gives a lot of the elderly physical and mental health Bring about disturbance, poor quality of long-term sleep can 
make people's physiological rhythm, brain function and other disorders, reduced mobility, the body caused a variety of hazards, seriously affecting physical and mental health. Studies have also shown that insomnia than the normal people have more depression and anxiety [2].

Hospital elderly patients as a special group, is also often sleep disturbed by this problem, in the hospital treatment of other diseases while taking sleeping pills to improve sleep is more common [3]. However, due to physical illness in hospitalized elderly patients, sleep quality problems are not easily cause medical attention. Only by understanding the mastered the elderly inpatient sleep and its influencing factors in order to provide more targeted interventions to facilitate the patient to maintain good physical and mental condition. Which improve the quality of sleep and efficacy of patients is also important. Therefore, the quality of sleep and influencing factors of elderly inpatients were investigated by means of questionnaires and reference literatures, so as to provide constructive intervention measures.

\section{Materials and Methods}

\subsection{Clinical data}

Age from 60 to 79 years, 169 cases, 80 years and above 102 cases; 112 cases of women, 118 males and 11 females; aged 60 years and above were admitted to the Department of Geriatrics, Yan'an Hospital from March to September, Select the primary disease with high blood pressure, diabetes, chronic bronchitis, emphysema, coronary heart disease and so on, no language and mental retardation patients, hyperthyroidism, cerebrovascular disease and long-term insomnia patients outside the hospital.

\subsection{Research tools}

The questionnaire was divided into 2 parts, (1) general information. (4) The sleep status self-rating scale (SRSS) was used to evaluate. The designated staff is responsible for the explanation before filling in. Each factor according to the frequency and severity of the problem in a 5-point system that a total score of 50 points, $\geqslant 23$ points were insomnia, the higher the score the more serious the problem of insomnia. Questionnaires are in the nursing staff under the guidance of the patient or family to help fill out.

\subsection{Results analysis}

All the data in this study were processed by SPSS 19.0. The measurement data were expressed as mean \pm standard deviation, and the percentages of count data $(\%)$ were used. Comparison of two sets of measurement data using t test to test the existence of statistical differences. 


\section{Result}

\subsection{General situation}

The questionnaires were issued a total of 262 , recycling $230,87.8 \%$ recovery rate. Among the respondents, there were 89 cases in junior college or above, 141 cases in senior high school and below; 132 cases of spouses and 98 cases of spouses; 102 cases of first hospitalization and 128 cases of repeated hospitalizations (refer to hospitalization $\geqslant 2$ times).

There were no significant differences in the scores of nightmare and night sleep among the elderly inpatients. Compared with the norm, the scores and scores of the other sleep scores were significantly higher than those of the national norm $(\mathrm{P}<0.01)$. This group of 230 cases of elderly patients with total sleep $\geqslant 23$ points accounted for $96 \%$, significantly higher than the norm $(45.60 \%)$ [4], the difference was significant, indicating that elderly patients are more likely to occur than normal insomnia.

Table 1230 patients SRSS score and norm comparison

\begin{tabular}{|c|c|c|c|c|}
\hline $\begin{array}{l}\text { Scoring } \\
\text { factor }\end{array}$ & $\begin{array}{c}\text { Norm } \\
(\mathrm{n}=13273)\end{array}$ & $\begin{array}{l}\text { This group } \\
(n=230)\end{array}$ & $(\mathrm{t})$ & $\mathrm{P}$ value \\
\hline lack of sleep & $2.80 \pm 0.87$ & $3.15 \pm 1.28$ & 4.17 & $<0.01$ \\
\hline lack of sleep & $2.80 \pm 0.87$ & $3.15 \pm 1.28$ & 4.17 & $<0.01$ \\
\hline Sleep quality & $2.33 \pm 0.81$ & $3.03 \pm 1.30$ & 8.11 & $<0.05$ \\
\hline $\begin{array}{l}\text { Lack of } \\
\text { arousal }\end{array}$ & $2.43 \pm 1.07$ & $2.91 \pm 1.16$ & 6.24 & $<0.01$ \\
\hline sleeping time & $2.30 \pm 0.63$ & $2.95 \pm 1.19$ & 8.28 & $<0.01$ \\
\hline $\begin{array}{c}\text { Difficulty } \\
\text { falling asleep }\end{array}$ & $1.98 \pm 0.99$ & $2.96 \pm 1.07$ & 13.89 & $<0.01$ \\
\hline $\begin{array}{c}\text { Sleep } \\
\text { instability }\end{array}$ & $2.00 \pm 1.01$ & $2.95 \pm 1.19$ & 12.02 & $<0.01$ \\
\hline $\begin{array}{l}\text { Morning } \\
\text { wake up }\end{array}$ & $2.00 \pm 1.05$ & $3.06 \pm 1.14$ & 13.99 & $<0.01$ \\
\hline Nightmare & $2.07 \pm 1.11$ & $2.25 \pm 1.31$ & 9.85 & \\
\hline $\begin{array}{c}\text { Medication } \\
\text { situation }\end{array}$ & $1.24 \pm 0.64$ & $3.02 \pm 1.07$ & 25.12 & $<0.01$ \\
\hline Eye loss after & $2.96 \pm 1.45$ & $3.29 \pm 1.32$ & 3.80 & $<0.01$ \\
\hline
\end{tabular}




\begin{tabular}{|c|c|c|c|c|}
\hline reaction & & & & \\
\hline Total score & $22.14 \pm 5.48$ & $30.24 \pm 3.83$ & 32.03 & $<0.01$ \\
\hline
\end{tabular}

\subsection{Comparison of sleep factor scores}

It can be seen from Table 2 that the male factor 3 was significantly higher than female patients $(\mathrm{P}<0.05)$, more prone to arousal, and female patients were $5,6,8,10$ points were significantly higher than male patients $\mathrm{P}<0.05)$. Spouses of patients with spouses were equally divided into 2, 3, 5, 6, 10 patients (all $\mathrm{P}$ $<0.05)$. $(\mathrm{P}<0.05)$.Conclusion The hospitalization rate of primary hospitalization is higher than that of repeat hospitalization $(\mathrm{P}<0.05)$.

The group of 230 patients, 225 cases of insomnia, the sleep status of the factors in Table 3, in which pathological factors, psychological problems, environmental and lifestyle changes for the three most important factors, accounting for $84 \%$, $75 \%$ and 70 / RTI \& gt; Other factors were environmental and lifestyle changes, sleep reversal, treatment and inappropriate stimulation, decreased activity, adverse drug reactions, personal bad habits.

\begin{tabular}{lcc}
\hline \multicolumn{1}{l}{ relevant factor } & Cases & percentage (\%) \\
\hline Pathological factors & 189 & 84 \\
Psychological problems & 168 & 75 \\
Environment and & 157 & 70 \\
lifestyle changes & 118 & 50 \\
Sleep upside down & 99 & 40 \\
Treatment $\quad$ and & 67 & 34 \\
inappropriate stimulation & 41 & 21 \\
Activity decreased & 32 & 16 \\
Adverse Drug Reactions & & \\
Personal bad habits & & \\
\hline
\end{tabular}

\section{Discuss}

\subsection{Insomnia is a common problem in elderly inpatients}

In this paper, the use of SRS prepared by Li and other 230 cases of elderly patients in the sleeping state of a sample survey, the results showed that the elderly inpatients than normal more prone to insomnia, the existence of sleep problems in addition to "Nightmare" $(\mathrm{P}<0.01)$, suggesting that the quality and quantity of sleep in the group there are more problems, we can see that insomnia 
has become a common health problems in elderly patients, it is particularly important to assess the importance.

\subsection{Elderly inpatient insomnia characteristics}

After investigation found that the elderly inpatient insomnia has the following characteristics: (1) elderly inpatients than normal people are more prone to insomnia, the quality and quantity of sleep in the group there are more problems, especially difficulty falling asleep, sleep instability and insomnia After the reaction of three aspects. (2) elderly patients without spouses more elderly spouses of emotional vulnerability, more prone to insomnia. (3) older female patients with initial hospitalization patients have more insomnia problems. The former with its reduced activity during the day, hi bed, good tea and sleep time, sleep shallow, vulnerable to interference from the external environment, coupled with their poor physical condition; female patients may be sensitive to their emotions, delicate, More concerned about; and for the initial hospitalization, may be with the strangeness of the environment and the first time facing the threat of disease, psychological temporarily difficult to adjust the relevant.

\subsection{The main factors leading to insomnia}

The most common cause of insomnia in elderly patients is the physical discomfort caused by the disease, including pain, dyspnea, palpitation, urinary frequency, cough, nausea, abdominal distension, physical braking and skin itching, the body in an uncomfortable state, Thus affecting sleep. Therefore, we should change the concept, should not be considered a "normal phenomenon" and ask patients to "tolerance", should seriously listen to the patient complain, careful observation of the patient's response, as long as the impact of sleep, contact with the doctor, be positive and effective symptomatic deal with. The second reason is the patient's psychological factors. Due to the pressure of work and life, the younger generation is often difficult to take into account the elderly [6], patients with varying degrees of psychological stress, such as fear of disease prognosis, illness and hospital expenses, can lead to bad emotional reactions. Loneliness, depression, fear, etc. [7]; therefore, when such patients insomnia, it is necessary to try to understand their psychological status, the reasons for their insomnia to psychological counseling, To strengthen the knowledge of disease-related missionary, if necessary, can fully mobilize their family social support system to provide emotional support. The third reason is the change in environmental habits. Including strange new environment, ward noise and monotonous life, so we should sleep time for the patient to provide a quiet rest environment, the treatment and care operations as far as possible one-time finish, for the noise source to take appropriate measures. At the same time the use of psychological therapy, behavior correction, sleep hygiene guidance and drug-assisted treatment combined with a variety of measures to intervene, can receive good results [8]. 


\section{Acknowledgement}

Shaanxi Province Health and Family Planning Commission 2016 Annual Health Research Project (2016D073)

Shaanxi Province Department of Education 2016 annual scientific research project (16JK1867)

Yan'an University 2015 annual university research project (YDK2015-70)

\section{References}

[1] Tian Yongjun, Miao Jingya. Orthopedics inpatients with insomnia-related factors. Chinese Journal of Nursing, 36 (3), pp. 227-289, 2001

[2] Li Li, Wang Liying, Li Min, et al Depression of elderly hospitalized patients and their relationship with physical disease. Chinese Journal of Geriatrics, 21 (3), pp. 221-222.2002, 21 (3), 221-222.

[3] Dai Fu-min, Kong Sha, Yang Yujie. Elderly patients with sleep status survey and implementation of the effect of nursing interventions. Journal of Practical Nursing, 11, pp.20-21.2002.

[4] Li Jianming, YIN Su-feng, DUAN Jian-xun, et al. Assessment of Sleep Status in 13273 Normal Persons by SRSS. Journal of Health Psychology, 8 (3), pp.351353.2000 .

[5] Li Jianming, Yin Sufeng, Duan Jianxun, et al. Self-Rating Sleep Status Inventory (SRSS). Journal of Health Psychology, 8 (3), pp.353-354.2000.

[6] Huirong, Zhang Huarui, Zhang Ruying. Evaluation of the health status of the elderly in China, Journal of Practical Nursing, 7, pp. 57-58, 2002.

[7] Kang Liyan, Diao Huimin, Zhang Lixin, etc. Inpatients psychological response factors and nursing strategies, Nursing Journal, 5, pp.383-384.2003.

[8] Zhao Xixi, Zhang Jun, Tan Chunying .Subjective nursing intervention on insomnia patients sleep. Chinese Journal of Nursing, 36 (12), pp. 923-924. 2001. 